\title{
Simulation of 4K UHDTV via Satellite Link
}

\author{
Neel Rohit \\ ${ }^{1}$ Sikkim Manipal Institute of Technology, Majhitar, East Sikkim-737136
}

\begin{abstract}
The prospect of $4 \mathrm{~K}$ UHDTV grows over the next few years, operators and service providers will need to begin to recognize the importance of delivering the next-generation television experience that consumers demand. In this experiments, only two service scenarios with single band and single codecs with one of AVC and HEVC schemes are applied. With increased screen size resulting in a larger viewed angle, for identically shot content, the angular velocity of motion for UHDTV is greater than for HD. As a result, motion is more perceivable as discrete steps in position. Frame rate increases can reduce this effect, however does increase both baseband and compressed video bit rates. UHDTV operates at 50.0 or $59.94 \mathrm{~Hz}$ progressive frame rates for video content, and 24.0 or $23.976 \mathrm{~Hz}$ progressive frame rates for native movie content (e.g. any UHDTV successor to BluRay).Colloquially, the video formats are often known as $4 \mathrm{Kp} 50$ or $4 \mathrm{Kp} 59.94$. These equate to $4 \mathrm{x}$ the $3 \mathrm{G}-\mathrm{SDI}$ line rates, so approximately $12 \mathrm{Gbit} / \mathrm{s}$.
\end{abstract}

Keywords: 4K, UHD, frame, baseband, bitrate, single band, single codecs, broadcast.

\section{Introduction}

The project describes the detailed broadcast system Description of the satellite link of UHDTV. It also gives the detailed explanation of the improvements over the existing broadcast technology.As screens become larger, the proportion of the overall human viewing angle occupied by the image is increased. One result of this is that the camera angle used to film the content should take into consideration the viewing angle. With UHDTV, it is possible to film with wider angle lenses, representing a closer match to the consumer's viewing angle for a large television. UHDTV's increased resolution allows this format change whilst keeping the resolvable detail. The end result is a more natural appearance to the images on the television. Owing to this condition it is the need of the day to develop the new broadcast technology that brings the transmission and reception direct from the satellite link.

4K UHD satellite experimental broadcasting services through the Ka band satellite have been conducted in past years. In this experiments, only two service scenarios with single band and single codecs with one of AVC and HEVC schemes are applied. The experiments were conducted to evaluate the multi-channel service provisioning with channel adaptability for $4 \mathrm{~K}$ UHD satellite broadcasting. To meet the requirement, a high-speed DVB-S2 modem was developed using VCM mode support. Most of the legacy DVBS2 modems are developed to support $\mathrm{Ku}$ band transponders of around $30 \mathrm{MHz}$ providing $80 \mathrm{Mbps}$ of maximum capacity. Our new modem extends this capacity upto around 300Mbps to support wideband $\mathrm{Ka}$ band transponders, which are required to provide multiple UHDTV channel services.

\section{The Ultra High Definition}

Ultra-high-definition television (also known as Super HiVision, Ultra HD television, UltraHD, UHDTV, or UHD) includes 4K UHD (2160p) and 8K UHD (4320p), which are two digital video formats proposed by NHK Science \& Technology Research Laboratories and defined and approved by the International Telecommunication Union (ITU). The Consumer Electronics Association announced on
October 17, 2012, that "Ultra High Definition", or "Ultra HD", would be used for displays that have an aspect ratio of 16:9 and at least one digital input capable of carrying and presenting native video at a minimum resolution of $3840 \times 2160$ pixels[1].

The human visual system has a limited ability to discern improvements in resolution below a certain size and beyond a certain distance. At current consumer home viewing distances and TV sizes, increasing resolution to $4 \mathrm{~K}$ may have little perceptual impact, as consumers would not be close enough (the Lechner distance) to appreciate the differences in pixel count between $4 \mathrm{~K}$ and HD. UHDTV also allows other image enhancements in dynamic range and color, which can improve the perceived difference between 4KTV and HDTV. UHDTV allows the future use of the new Rec. 2020 (UHDTV) color space which can reproduce colors that cannot be shown with the current Rec. 709(HDTV and most current 4KTV) color space. When dealing with CIE 1931 color space coverage, the Rec. 2020 color space covers $75.8 \%$, whereas the digital cinema reference projector color space covers 53.6\%, the Adobe RGB color space covers 52.1\%, and the Rec. 709 color space covers $35.9 \%$.UHDTV also allows for an increase in dynamic range, meaning brighter highlights but also increased detail in the greyscale. UHDTV also allows for frame rates up to 120 frames per second (fps). Note that UHDTV potentially allows Rec.2020, higher dynamic range and higher frame rates to be applied to HD services, without necessarily increasing resolution to $4 \mathrm{~K}$.

\section{The 4K System}

\subsection{Performance}

The DVB-S2 standard has been specified to meet the demands for the best transmission performance, total flexibility, and reasonable receiver complexity. To achieve the best performance-complexity trade-off, DVB-S2 benefits from more recent developments in channel coding and modulation. Moreover, the system was intended to be used for several applications including interactive point-to-point applications and professional applications, such as IP 


\section{International Journal of Science and Research (IJSR) \\ ISSN (Online): 2319-7064}

Index Copernicus Value (2013): 6.14 | Impact Factor (2015): 6.391

unicasting, digital TV, news gathering, and data content distribution. The unique features making these diverse applications achievable is the adoption of adaptive coding and modulation (ACM) functionality, which allows an optimization of the transmission parameters for each individual user on a frame-by-frame basis depending on the path conditions under closed-loop control through a return channel. To keep the packet error rate at less than $10^{\wedge}-7$ over an AWGN channel, it is known that the required $\mathrm{C} / \mathrm{N}$ of the DVB-S2 system varies from $-2.4 \mathrm{~dB}$ with QPSK $1 / 4$ to $16 \mathrm{~dB}$ with 32APSK 9/10. However, since the effects of nonlinearity and synchronization loss and phase noise should be considered along with the ideal performance, we take 0.6/1.0/2.0/4.0 $\mathrm{dB}$ as an additional power losses for QPSK/8PSK/16APSK/32APSK, as reasonable estimates with typical equipment characteristics, respectively. Therefore, the $\mathrm{C} / \mathrm{N}$ values required to meet $10^{\wedge-7}$ of PER over an AWGN channel for each modulation.

ITU has recently defined two specific formats for use in UHDTV systems:

UHDTV Level 1 Resolution $=3840 \times 2160$

UHDTV Level 2 Resolution $=7680 \times 4320$

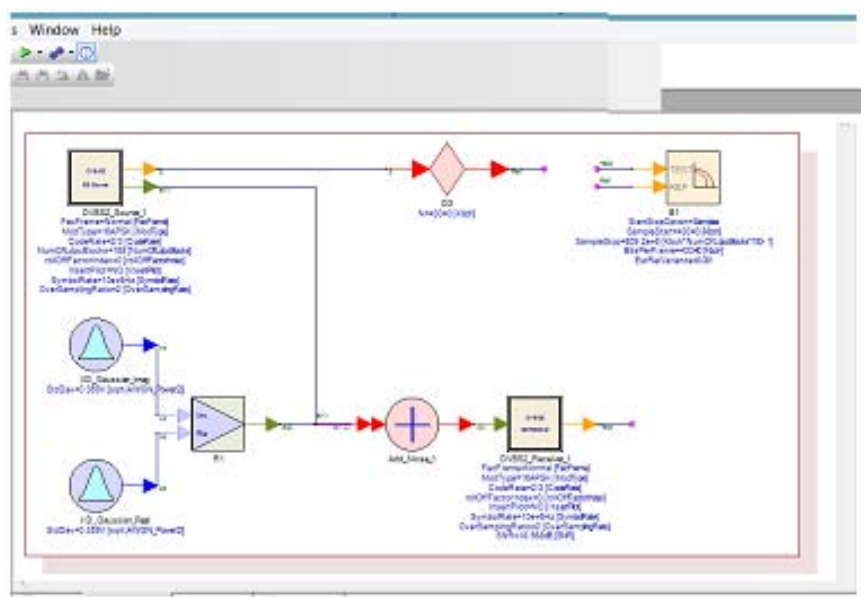

Figure 1: SystemVue Model for DVBS2 System.

\subsection{Table}

Table 1: Signal level and quality for frequency levels

\begin{tabular}{|c|c|c|c|c|}
\hline $\begin{array}{c}\text { Carrier } \\
\text { Frequency Fc }\end{array}$ & $\begin{array}{c}\text { Symbol Rate } \\
\text { (KSymbols/sec) }\end{array}$ & $\begin{array}{c}\text { No. Of } \\
\text { Samples } \\
\text { Collected }\end{array}$ & $\begin{array}{c}\text { Signal } \\
\text { Quality }\end{array}$ & $\begin{array}{c}\text { Signal } \\
\text { Level }\end{array}$ \\
\hline $1.422 \mathrm{GHz}$ & 30000 & 59940001 & $64 \%$ & 14 \\
\hline $1.422 \mathrm{GHz}$ & 32000 & 63936001 & $82 \%$ & 21 \\
\hline $1.422 \mathrm{GHz}$ & 34000 & 67932001 & $99 \%$ & 22 \\
\hline $1.422 \mathrm{GHz}$ & 36000 & 71928001 & $99 \%$ & 22 \\
\hline $1.422 \mathrm{GHz}$ & 45000 & 89910001 & $99 \%$ & 22 \\
\hline
\end{tabular}

\subsection{HEVC-NEW CODING STANDARD}

In January 2013, version 1 of High Efficiency Video Coding ("HEVC") was approved as a final draft international standard, acquiring the designation H.265/ISO23009-1 as a result. HEVC offers bit rate efficiency improvements over H.264 of $35-50 \%$ depending on content, resolution, operating point, etc. In particular, the largest efficiency improvements are found at higher resolutions -not surprisingly because HEVC introduced tools that were specifically targeted at increased picture resolutions (recognizing that 16x16 macro blocks were designed for content at around SD resolution).The use of HEVC means that it would be realistic to reach a point where a UHDTV service can be transmitted in the same bandwidth as an MPEG-2 HD service is today.

More options will exist for decoding HEVC UHDTV in the home than was previously the case at the launch of HD H.264. STB silicon can reasonably be expected to be in place to allow services to be on air in time for the Rio 2016 Olympics, if not before. Next-generation games consoles are likely to have decode capability that would allow them to be used as alternative decode devices - and it is likely that they will form a platform capable of decoding the next-generation disc formats for movie content. Both of these, of course, are dependent on the standardization and deployment of an updated HDMI specification that is capable of more than 30 frames per second.

Version 1 of HEVC includes both Main and Main 10 profiles. The only key difference between these profiles is the bit depth, Main 10 allows 10 bits per pixel, whereas Main only allows 8 bits. It is likely that Main (i.e. 8-bit) will be used on content targeted at tablets, mobile phones, etc, however one of the limiting factors for the consumer experience today with HD is the 8-bit resolution. This leads to contouring, most noticeable on content with slowly changing levels across a picture, particularly when associated with low levels of chrominance.

The Main10 profile addresses this by including 10-bit resolution as an option for Main10 capable decoders, thus providing an improved viewing experience - considered a key motivation for UHDTV television. As a side effect of the likely difference between different devices' likely support for Main10, it is likely that a television connected to a Main10 capable STB or console will be the highest quality way to view content in UHDTV.For a 1080-line HD screen, the resolution limit is reached when the viewing distance is approximately $1.6 \mathrm{x}$ the diagonal screen size. UHDTV Level 1 shows visible benefits over 1080- line HD at all distances closer than $1.6 \mathrm{x}$ diagonal, and reaches the limit of perceivable resolution at approximately $0.75 \mathrm{x}$ the diagonal size. Recent UHDTV Level 1 TVs are typically around 84" diagonal. At this size: UHDTV Level 1 provides an improved viewer experience at distances closer than approximately $3.4 \mathrm{~m}$, progressively more visible until

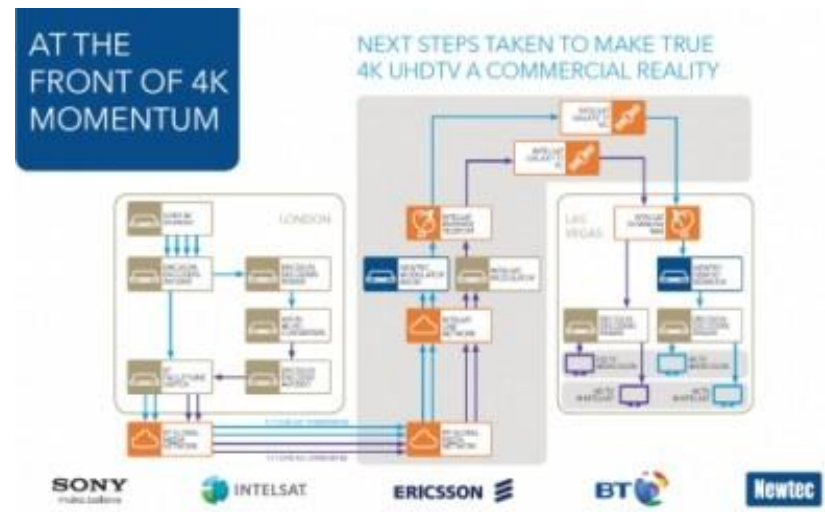

Figure 2: Satellite Transponder Link of UHDTV 


\section{International Journal of Science and Research (IJSR) \\ ISSN (Online): 2319-7064}

Index Copernicus Value (2013): 6.14 | Impact Factor (2015): 6.391 3.4 Analysis of the modulation techniques used for
UHDTV broadcast in Korea

Since its inception with a 24 hours-a-day single channel service in 2003, digital HDTV satellite broadcasting service in Korea has developed into a large market with more than 100 HDTV channels and about four-million subscribers. Currently, digital satellite broadcasting services are provided through the Ku-band transponder of the KoreaSAT-6 satellite, which was launched in December 2010, and they will be extended for next-generation broadcasting services, such as stereoscopic 3-Dimentional TV (3DTV) and Ultra HDTV (UHDTV). These new broadcasting services will demand frequency capacity more than the current saturated Ku-band, and to resolve such limitations, the 21.4 to 22.0 $\mathrm{GHz}$ frequency band was allocated for Broadcasting Satellite Service (BSS) in regions 1 and 3 at the World Administrative Radio Conference-92 (WARC-92) for implementation after April 1, 2007. According to this allocation, many countries in these regions have been competitively requesting frequency registration for this frequency band, and the number of registrations has increased significantly to up to 700 satellite networks. However, since propagation attenuations in this bandwidth may place a heavy restriction on the service availability and system feasibility, mitigation techniques have been studied from diverse perspectives. Many advanced countries have developed various Ultra High Definition (UHD) broadcasting technologies, opening a new horizon for the possibility of commercial UHD broadcasting service. Since it began its R\&D activities for UHD broadcasting service in 1995, Japan has established a new concept for nextgeneration broadcasting service that fully satisfies the human perception capacity in a visual and auditory sense. With overall research results in the every part of broadcasting chain, the Japanese government announced the launch of trial broadcasting at $4 \mathrm{~K}$ resolution for mid-2014 and the start of test broadcasting at $8 \mathrm{~K}$ resolution for 2016. In the case of Korea, the pay TV operators conducted experiments for UHD broadcasting service and started their trial service from the first quarter of 2014. For this end, satellite transmission tests and terrestrial broadcasting tests for 4K UHD broadcasting service were successfully conducted in 2013. During the initial stage of such service, satellites are expected to be the major medium because of its flexibility and adaptability for new services. However, since the propagation attenuation in the Ka band, particularly from rain, poses a significant challenge in service availability, it is needed to investigate the pertinence and possibility of commercial broadcasting services using this band for immersive media including UHD video. Moreover, since the weather in Korea has been changing to an increase in rainfall in recent years, worsening the conditions for satellite broadcasting, reasonable service scenarios should be carefully taken into consideration. For this purpose, a simple analysis was conducted to identify what kinds of services are possible. In this paper, an analysis of previous rain attenuation models is conducted to confirm their results, and a link analysis is extended to consider more detailed parameters.

Through these analyses, much more diverse service scenarios are envisaged. The remainder of this paper is organized as follows. A rain attenuation analysis for UHD broadcasting using a satellite at the Ka frequency band, to identify how much rain attenuation has to be expected using the regional rain distribution of Korea, is presented in Section II. In addition, an analysis of the link margin for each code rate and the modulation method of DVB-S2 at a certain rain rate are presented to evaluate the service availability in Section III. Next, channel-adaptive UHD satellite broadcasting scenarios based on the combinations of various technical elements are provided in Section IV, and the experimental progress for UHD satellite broadcasting service conducted in Korea is described in Section V.

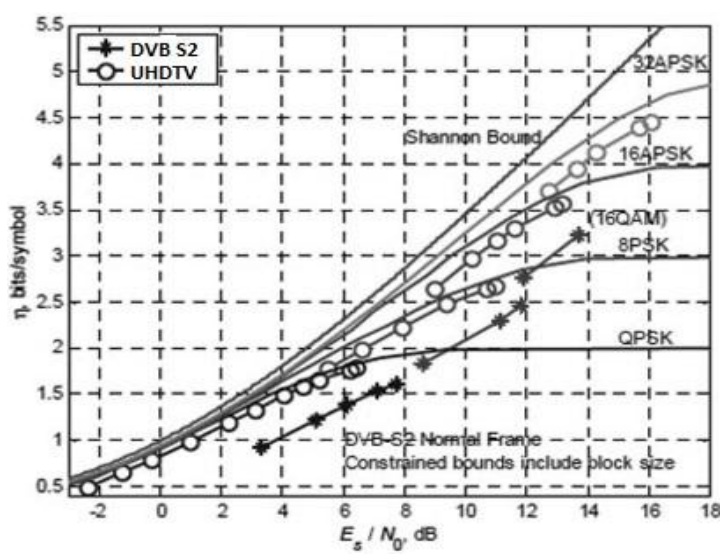

Figure 3: Comparison chart for DVBS2 and $4 \mathrm{~K}$

\subsection{Problem Definition}

The $4 \mathrm{~K}$ broadcast is nothing but an improved version of the older $2 \mathrm{~K}$ broadcasting technique. It is known that all it has that the symbol rate has to be increased from the standard parameters. The rates required for the $4 \mathrm{~K}$ modulation is 10 times the higher bandwidth compared to the present DVBS2 system. But achieving so high a bandwidth is out of the scope of the infrastructure. The traditional DVBS2 modulation can work up to the frequency of $30 \mathrm{MHz}$. Our infrastructure gives us the capability to exceed it to $45 \mathrm{MHz}$.

But, it was found that just increasing the symbol rate doesn't help. Beyond the breakpoint of $36000 \mathrm{KSymbols/sec} \mathrm{there} \mathrm{is}$ heavy fluctuation. It is the need ultimately to clear off the fluctuations to get the reception very stable for reception.

The problems associated with the broadcasting technique of UHDTV is the Rain Attenuation phenomenon. As per the present scenario the channel adaptability is very less compared to the requirements to overcome the rain effects on the signal quality, BER value and strength. This reduces the throughput value drastically as the Doppler Shift mechanism infects the transmission signal with an additional shift frequency. As a result the overall error rate increases by more than $10 \%$.

The frame rate for a particular UHDTV is more than $60 \mathrm{~Hz}$.Thus it requires more bandwidth. With the increase in number of users, it becomes difficult to just work at a limitation of $21.4 \mathrm{GHz}$ to $22.0 \mathrm{GHz}$ frequency which was allocated for Broadcasting Satellite Service (BSS).

\section{Volume 5 Issue 4, April 2016




\section{Mathematical Equation}

Our studies suggest that an increase in symbol rate works well except for the condition that it has to be greater than the conventional level of Nyquist Sampling Rate. That is the sampling rate has to be increased by 3 times or 4 times. We observed that there is an increase in stability of the system. The broadcasting of the system is seen to be far more stable. The heavy fluctuations are removed to some extent due to this oversampling. Trials have been made to be done upto 3 times the sampling rate. Further stability can be achieved using the higher oversampling ratios.

The Nyquist Sampling Rate Equation is defined by:

$$
\mathrm{F}_{\mathrm{c}} \geq 2 \mathrm{~F}_{\mathrm{m}}
$$

Where,

$\mathrm{F}_{\mathrm{C}}$ is the carrier frequency and,

$\mathrm{F}_{\mathrm{m}}$ is the message signal frequency.

Another mathematical Equation used in this entire project was the Shannon's Channel Capacity Theorem, which is defined by:

$$
\mathrm{C}=\mathrm{B} \log _{2}(1+\mathrm{SNR})
$$

Where,

$\mathrm{C}=$ Channel Capacity,

$\mathrm{B}=$ Bandwidth and,

SNR=Signal to Noise Ratio.

\section{Future Prospective}

There are set top boxes that are being designed for giving data rates up to about $150 \mathrm{Msps}$. These boxes are generally used for satellite transponder uplink and downlink which are very costly for commercial purposes. IIT Madras has one of the models boxes known as Comtech CDM-760 high speed trunking modem. With such equipment also our purpose if fulfilled provided the commercialization is done and the cost is reduced.

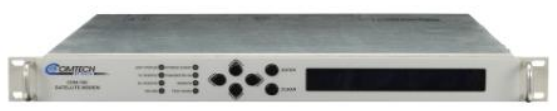

Figure 4: CDM-760 Modem

\section{Recommendations}

The project was conducted under the guidance of Dr. R.N. Bera, Head of the Department, Electronics and Communication Engineering, Sikkim Manipal Institute of Technology and Mr. Debasish Bhaskar, Assistant Professor, Electronics and Communication Engineering, Sikkim Manipal Institute of Technology.

\section{References}

[1] Advantech Wireless DVB-S2 Technical Presentation.
[2] UBICOMM 2014 : The Eighth International Conferenceon Mobile Ubiquitous Computing, Systems, Services and Technologies "A Study on the Ka-band Satellite 4K-UHD Broadcasting Service Provisioning in Korea", (2014)

[3] Ericsson UHDTV Acquisition and Exchange Whitepaper (2014).

[4] ETSI EN 302 307-1 Digital Video Broadcasting Part 1 (2014).

[5] ETSI EN 307 302-2 UHD Broadcasting Techniques (2015).

\section{Author Profile}

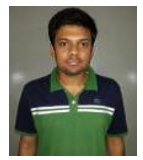

Neel Rohit, currently pursuing B.TECH from Sikkim Manipal Institute of Technology in Electronics and Communication Engineering (2012- 2016). 